\title{
The Thirty Meter Telescope (TMT): An International Observatory
}

\author{
Gary H. Sanders ${ }^{1,2}$ \\ ${ }^{1}$ Division of Physics, Mathematics and Astronomy, California Institute of Technology, \\ Pasadena, CA 91125, USA. \\ ${ }^{2}$ TMT Observatory Corporation, 1111 S. Arroyo Pkwy., Ste. 200, Pasadena, CA 91125, USA. \\ e-mail: sanders@tmt.org
}

Received 18 March 2013; accepted 6 May 2013

\begin{abstract}
The Thirty Meter Telescope (TMT) will be the first truly global ground-based optical/infrared observatory. It will initiate the era of extremely large (30-meter class) telescopes with diffraction limited performance from its vantage point in the northern hemisphere on Mauna Kea, Hawaii, USA. The astronomy communities of India, Canada, China, Japan and the USA are shaping its science goals, suite of instrumentation and the system design of the TMT observatory. With large and open Nasmyth-focus platforms for generations of science instruments, TMT will have the versatility and flexibility for its envisioned 50 years of forefront astronomy. The TMT design employs the filled-aperture finelysegmented primary mirror technology pioneered with the W.M. Keck 10-meter telescopes. With TMT's 492 segments optically phased, and by employing laser guide star assisted multi-conjugate adaptive optics, TMT will achieve the full diffraction limited performance of its 30-meter aperture, enabling unprecedented wide field imaging and multi-object spectroscopy. The TMT project is a global effort of its partners with all partners contributing to the design, technology development, construction and scientific use of the observatory. TMT will extend astronomy with extremely large telescopes to all of its global communities.
\end{abstract}

Key words. Optical astronomy-infrared astronomy-telescopeadaptive optics—-segmented mirrors.

\section{Introduction}

The Thirty Meter Telescope (TMT) will be the first truly global ground-based optical/infrared observatory. With the partnership of the astronomy communities, and their sponsors, of India, Canada, China, Japan and the USA, TMT represents a scale of global collaboration well beyond the previous 8-and 10-meter telescope projects in Chile and Hawaii. TMT will bring its scientific reach to the astronomy communities of a large fraction of the Earth's population, serving as a global intellectual asset. Towards that end, the astronomy communities of India, Canada, China, 
Japan and the USA are each shaping TMT's science goals, suite of instrumentation and the system design of the TMT observatory. In particular, the astronomy community of India carried out a detailed study of options (India's Participation in the Thirty Meter Telescope Project 2010) in choosing TMT as a principle element of its future program.

\section{The TMT site}

TMT will initiate the era of extremely large (30-meter class) telescopes with diffraction limited performance from its vantage point in the northern hemisphere on Mauna Kea, Hawaii, USA. Centered in the Pacific Ocean, amidst its astronomy stakeholders, Mauna Kea provides world leading astronomical seeing conditions (Schoeck et al. 2009). TMT will be installed on a plateau adjacent to the ridge upon which numerous other optical observatories (W.M. Keck Observatory, Gemini South, Subaru, Canada France Hawaii Telescope, and several others) are located. TMT is the only 30-meter class observatory planned for a northern hemisphere location.

In order to carry out construction and operations on Mauna Kea, an approved State of Hawaii Environmental Impact Statement must be prepared and approved. This approval was obtained by TMT in 2010. In addition, any construction and use of land on Mauna Kea requires issuance of a State of Hawaii Conservation District Use Permit, certifying that the proposed activity is consistent with the planned uses of the particular land and that the mountain's resources are being conserved. All of the required submittals, public hearings and administrative processes have been completed and TMT expects to receive this permit in 2013.

The planned start of TMT construction is April 2014, with technically-paced completion of the project enabling early science in late 2021. Towards that construction start, with legal access to the site expected soon, TMT has completed the first round of geotechnical studies of the site employing ground penetrating radar and multichannel analysis of sonic waves. These studies yielded sonic wave velocities and general information about strata, voids and structure. The design of the telescope pier is now informed by this information. Further complete geotechnical borings are planned as soon as legal right of entry is achieved.

\section{TMT science based requirements}

The TMT project was formally initiated in April, 2004 following three precursor design studies (Nelson 2002; Strom 2002; Roberts et al. 2002), and the TMT Science Advisory Committee (SAC) prepared a Science-based Requirements Document (SRD) (http://tmt.org/sites/default/files/SRD-CCR18-Science-Based-RequirementsDocument.pdf) that defined desired TMT science and the capabilities that the TMT observatory was required to have in order to accomplish these science programs. The science programs studied were described more fully in a Detailed Science Case (DSC) (http://tmt.org/sites/default/files/TMT-DSC-2007-R1.pdf) document.

The SRD capabilities were largely packaged into instrument concepts that were used, in 2005-2006, to support a number of studies, by independent astronomy groups, of feasible instruments that would satisfy the SRD requirements. These 
diverse, but specific, instrument concepts enabled the development of a TMT Observatory Requirements Document (ORD) (http://tmt.org/sites/default/files/documents/ application/pdf/ord-ccr25.pdf) representing the highest level technical and engineering requirements for the TMT design. The starting point for the design was captured in an Observatory Architecture Document (OAD) (http://tmt.org/sites/default/ files/documents/application/pdf/oad-ccr25.pdf) that provides the accompanying design architecture that meets the requirements of the ORD.

The design staff of the TMT project is governed by the ORD and OAD in all TMT design development. These two documents represent the highest level of TMT system engineering. A crucial feature of TMT system engineering is frequent communication and interaction between the design team and the SAC so that the scientific impact of design tradeoffs, choices and study results are well understood and that the SAC is assured of having an important voice in assuring the delivery of TMT science capabilities. All TMT astronomy communities are represented through the SAC.

\section{TMT architecture}

Figure 1 shows a cutaway isometric view of the observatory and includes a description of the main features of the optical architecture. TMT is a Ritchey-Chretien 3mirror telescope delivering a 20' field of view. The primary mirror is a filled-aperture finely-segmented hyperboloid with 492 segments filling its 30 meter diameter. The convex secondary mirror is challenging to test optically but by choosing this arrangement over a Gregorian design, the $f / 1.0$ telescope is 6 meters shorter, reducing the size of the telescope, dome enclosure and the required site footprint. A key feature is the flat, fully articulated tertiary mirror that enables rapid changes from one instrument on the Nasmyth focus platforms to another. Thus, the observatory is able to respond quickly to transient phenomena, changes in atmospheric conditions or to observing programs that require agile use of several instruments.

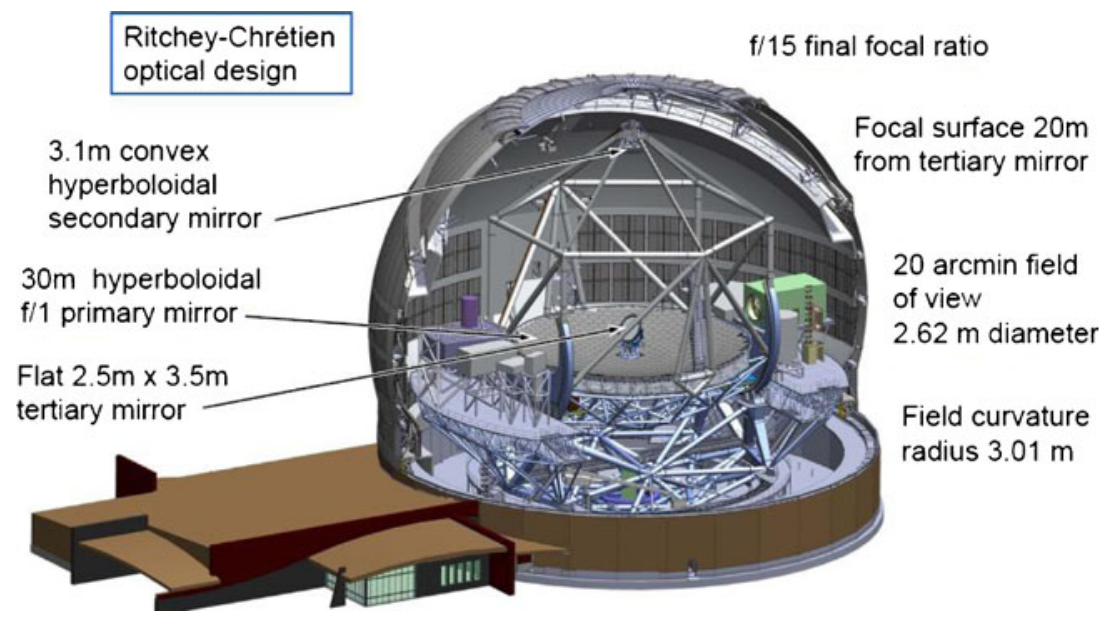

Figure 1. TMT observatory with principal optical features. 
The decision to place the telescope elevation axis above the primary mirror made it possible to employ the Nasmyth platforms and articulated tertiary mirror to gain such versatility. Furthermore, the large Nasmyth platforms can accommodate a large number of instruments at once or evolution of the instrument suite during the life of the observatory. Nasmyth platforms also provide a constant orientation for instruments as the telescope elevation angle varies from zenith to the observational limit of 65 degrees. The resulting constant gravitational vector force simplifies instrument opto-mechanical design. TMT's early instrument studies demonstrated that up to about 8 instruments may be accommodated at the same time (Simard 2013).

\section{TMT primary and its control systems}

Closely based upon the technology base pioneered by the segmented mirror twin 10-meter W.M. Keck Observatory telescopes, the TMT primary mirror extends the technology to a much larger segment count, 492 segments compared to Keck's 36 segments. This is primarily a manufacturing challenge in that reducing the per segment costs while maintaining optical quality is essential to such a large project. In order to satisfy this manufacturing goal, TMT is prototyping primary mirror segments at several locations in the TMT partnership including firms with prior experience in producing aspheric astronomy mirrors and new firms in partner countries. The goal is to develop robust and cost effective supply chains in the USA, Japan, China and India, with equivalent quality and conformance to TMT requirements. The most aspheric segment types, corresponding to the outer rings of the hyperboloidal mirror, have already been produced in the USA and Japan and prototyping is underway in China and planned in the future for India. Segments have also been successfully mounted, in the USA and Japan, on their support systems.

Control systems hardware such as segment support systems, on-segment edge sensors that enable segments to be located with respect to their neighbors, and segment actuators that respond to control system commands to adjust segment positions, will be produced in India. Already, edge sensor and actuator prototype parts have been fabricated in India and these are undergoing tests. Full segment support assemblies will be fabricated in India during 2013. The primary mirror control algorithms, and the alignment and phasing systems for the primary mirror, are being developed by TMT in California, at the University of California, Irvine and the Jet Propulsion Laboratory. These same teams were responsible for the corresponding systems at the W.M. Keck Observatory.

\section{Secondary and tertiary mirror systems}

As all control of the 3-mirror telescope wave front can be carried out with the primary mirror, the secondary and tertiary mirrors can be entirely passive systems. Both employ passive 18-point whiffle tree supports. They have been the subject of 7 industrial studies that defined design concepts and costs for constructing these systems. The secondary mirror is planned for fabrication by the USA partners. The Changchun Institute of Optics, Fine Mechanics and Physics (CIOMP), China will provide the tertiary mirror system which is also referred to as a giant science steering 
mirror as its primary function is to place the telescope optical beam on the selected science instrument.

\section{Adaptive optics and instrumentation}

Reflecting the scientific interests of all TMT partner communities, all partners are involved in instrumentation, including the adaptive optics systems. TMT adaptive optics is being designed and prototyped in efforts in the Canada, China and the USA (Ellerbroek 2013). TMT science instruments involve groups in all partner countries.

The main facility adaptive optics system, NFIRAOS, which can support three instruments at the same time, is being designed at the National Research Council Laboratory in Victoria, BC, Canada. Deformable mirror development for this multiconjugate system is being led by the project office with significant prototyping and testing underway. Polar coordinate and conventional wave front sensors are being prototyped and tested in the USA. Lasers to provide artificial guide stars, using the atmospheric sodium ion layer, are being prototyped by the project with a commercial supplier and also by the Chinese TMT partners at the Technical Institute of Physics and Chemistry in Beijing, China. The Chinese effort has already included two on-sky tests of the guide star system performance. The system to transport the light from the guide star lasers mounted at the TMT elevation axis to the top end of the telescope for launch is under design by the Institute of Optics and Electrics, part of TMT's China consortium. Dubbed the Laser Guide Star Facility, the system will provide 6 guide stars in a constellation (asterism) for use at first light, and 9 stars later in TMT operations.

\section{Observatory software}

TMT observatory software, including common services, observatory control, user interfaces, data acquisition and archiving and observation planning, will be an effort of India and the USA partners. FOVAST, A Focal Plane Visualization and Asterism Selection Tool has been prototyped in India to support control of science instruments during observations. In addition, an infrared guide star catalog study is underway in India (Subramanian et al. 2013) and a design study of TMT event services, also in India, is planned later in 2013.

\section{Conclusions}

TMT will open the era of extremely large telescope observing with diffraction limited performance to generations of observation from Mauna Kea. However, it also represents an unprecedented global partnership in astronomy planning, telescope technology development, big science collaboration and project management and, most important, it initiates a new era in which frontier optical and infrared astronomy will be carried out by the national and international communities of Asia and America. Beyond its enormous light collection capability and the power of its resolution, TMT's science reach will focus the human capital, the talent and curiosity, of much of the global community. 


\section{Acknowledgements}

The TMT Project gratefully acknowledges the support of the TMT collaborating institutions. They are the Association of Canadian Universities for Research in Astronomy (ACURA), the California Institute of Technology, the University of California, the National Astronomical Observatory of Japan, the National Astronomical Observatories of China and their consortium partners, and the Department of Science and Technology of India and their supported institutes. This work was supported as well by the Gordon and Betty Moore Foundation, the Canada Foundation for Innovation, the Ontario Ministry of Research and Innovation, the National Research Council of Canada, the Natural Sciences and Engineering Research Council of Canada, the British Columbia Knowledge Development Fund, the Association of Universities for Research in Astronomy (AURA) and the U.S. National Science Foundation.

\section{References}

Ellerbroek, B. L. 2013, A status report on the Thirty Meter Telescope adaptive optics program, $J A A, \mathbf{3 4}(2), 121-139$.

http://tmt.org/sites/default/files/SRD-CCR18-Science-Based-Requirements-Document.pdf. This reference links to the current version of the SRD, originally authored in 2004 by the North American partners in TMT and subsequently revised by the expanded TMT Science Advisory Committee as the TMT collaboration grew to include the communities of India, China and Japan.

http://tmt.org/sites/default/files/TMT-DSC-2007-R1.pdf. This document, like the SRD, has been maintained and developed during the course of TMT design development.

http://tmt.org/sites/default/files/documents/application/pdf/oad-ccr25.pdf. This link is to the current approved version.

http://tmt.org/sites/default/files/documents/application/pdf/ord-ccr25.pdf. This link is to the current approved version.

India's Participation in the Thirty Meter Telescope Project: Proposal for Technology Capability Development and Demonstration, submitted to the Department of Science and Technology, Government of India, November 2010.

Nelson, J. E. 2002, Progress on the California Extremely Large Telescope (CELT), Proceedings of the SPIE, 4840, 47-59.

Roberts, S. C., Morbey, C. L., Crabtree, D. R., Carlberg, R., Crampton, D., Davidge, T. J., Fitzsimmons, J. T., Gedig, M. H., Halliday, D. J., Hesser, J. E., Herriot, G., Oke, J. B., Pazder, J. S., Szeto, K., Veran, J.-P. 2002, Canadian very large optical telescope technical studies, Proceedings of the SPIE, 4840, 104-115.

Schoeck, M. et al. 2009, Thirty Meter Telescope Site Testing I: Overview, PASP, 121-878, 384-395.

Simard, L. 2013, The Thirty Meter Telescope: Science and Instrumentation for a NextGeneration Observatory, JAA, 34(2), 97-120.

Strom, S. E. 2002, Giant Segmented Mirror Telescope: a point design based on science drivers, Proceedings of the SPIE, 4840, 93-103.

Subramanian, S., Subramanian, A., Simard, L., Gillies, K., Ramaprakash, A. N., Anupama, G. C., Stalin, C. S., Ravindranath, S., Reddy, B. E. 2013, Report on the Phase I of TMT Infrared Guide Star Catalog (TMT IR GSC), TMT.SFT.TEC.12.017.DRF06. 